\title{
Search for Higgs shifts in white dwarfs
}

\author{
Roberto Onofrio ${ }^{1,2}$ and Gary A. Wegner ${ }^{3}$
}

\begin{abstract}
We report on a search for differential shifts between electronic and vibronic transitions in carbon-rich white dwarfs BPM 27606 and Procyon B. The absence of differential shifts within the spectral resolution and taking into account systematic effects such as space motion and pressure shifts allows us to set the first upper bound of astrophysical origin on the coupling between the Higgs field and the Kreschmann curvature invariant. Our analysis provides the basis for a more general methodology to derive bounds to the coupling of long-range scalar fields to curvature invariants in an astrophysical setting complementary to the ones available from high-energy physics or table-top experiments.
\end{abstract}

Subject headings: atomic processes — gravitation — white dwarfs

\section{Introduction}

The recent discovery at the Large Hadron Collider (LHC) of a resonance at $125 \mathrm{GeV}$ compatible with the expectations for the Higgs particle (Chatrchyan et al. 2012; Aaad et al. 2012) represents a major step towards understanding the origin of the mass of fundamental particles. Eventually, this should also affect the other subfield in which mass has a pivotal role, i.e. gravitation. This is particularly relevant in models in which the Higgs field has nonminimal coupling to the general relativity sector, as invoked in various extensions of the standard model. Nonminimal coupling between the Higgs and spacetime curvature may be beneficial to have the Higgs responsible for inflation (Bezrukov \& Shaposhnikov 2008; Bezrukov et al. 2009), and as a suppression mechanism for the contribution to dark

\footnotetext{
${ }^{1}$ Dipartimento di Fisica e Astronomia "Galileo Galilei," Università di Padova, Via Marzolo 8, 35131 Padova, Italy; onofrior@gmail.com

${ }^{2}$ ITAMP, Harvard-Smithsonian Center for Astrophysics, Cambridge, MA 02138, USA

${ }^{3}$ Department of Physics and Astronomy, Dartmouth College, 6127 Wilder Laboratory, Hanover, NH 03755, USA; gary.a.wegner@dartmouth.edu
} 
energy expected from quantum fields (Shapiro \& Solà 2000). Upper bounds on the gravitational interaction of Higgs bosons from the LHC experiments have been recently discussed (Atkins \& Calmet 2013; Xianyu et al. 2013).

Bounds on the crosstalk between the Higgs particle and gravity may also be obtained by considering strong-gravity astrophysical objects, as proposed in Onofrio (2010) in the case of active galactic nuclei (AGN) and primordial black holes. The presence of a strong spacetime curvature deforms the vacuum expectaction value of the Higgs field and therefore the mass of fundamental particles such as the electron. Nucleons instead should be minimally affected by the strong curvature since most of their mass arises from the gluonic fields that, being massless, are not coupled to the Higgs field at tree level. Peculiar wavelength shifts are therefore predicted which should be present for electronic transitions and strongly suppressed for molecular transitions in which the main role is played by the nuclei themselves, such as in vibrational or rotational spectroscopy. Due to the vanishing of the Ricci scalar for spherically symmetric objects, attention was focused on the possibility of couplings to the only non-null curvature invariant, the Kreschmann invariant, defined as $K=R_{\mu \nu \rho \sigma} R^{\mu \nu \rho \sigma}$, where $R^{\mu \nu \rho \sigma}$ is the Riemann curvature tensor. This invariant plays an important role in quadratic theories of gravity (Deser \& van Niewenhuizen 1974; Stelle 1977; Hehl et al. 1978), and more in general in modified $f(R)$ theories (Sotiriou \& Faraoni 2010) and Einstein-Gauss-Bonnet models of gravity (Lovelock 1971).

While AGNs would provide a strong-gravity setting near their black holes, their complex structure and the presence of turbulence and high-energy interactions near the accretion region induce uncontrollable systematic effects which hinder the possibility for extracting bounds on a Higgs-Kreschmann coupling as this relies upon the simultaneous observation of atomic and molecular transitions. To our knowledge, no neutron stars appear to show both molecular and atomic lines in their spectra, while white dwarfs have both. Although their surface gravity is much weaker than around AGNs and neutron stars, many features can be controlled more precisely, thus providing a 'quieter' environment to search for the putative Higgs shift.

White dwarfs have been known since the 19th century and in addition to their interest for astronomical and cosmological problems including understanding the late stages of stellar evolution, determining the galaxy's age, and the nature of Ia supernovae, they have had a prominent role in fundamental physics since the early 20th century. Adams (1925) made the first attempt to verify general relativity by measuring the gravitational redshift of Sirius B. Chandrasekhar (1935) studied the consequences of Fermi-Dirac statistics for stars, introducing his celebrated limit. Bounds on the distance dependence of the Newtonian gravitational constant have been discussed comparing observations and models for the white dwarf Sir- 
ius B (Hut 1981) and those in the Hyades (Wegner 1989). More recently Berengut et al. (2013) proposed using white dwarfs to study the dependence of the fine structure constant on gravity. Here we show that white dwarfs can be used to obtain limits on the coupling of the Higgs field to a specific curvature invariant, by means of spectroscopic observations of a carbon-rich white dwarf, BPM 27606, using the Southern African Large Telescope (SALT). The analysis is complemented by considering data taken from the HST archive on a second white dwarf, Procyon B, in which CaII and MgII lines, in addition to the $\mathrm{C}_{2}$ bands, are also present.

\section{Higgs-shifts and Kreschmann invariant}

The search for coupling between the Higgs (or any scalar field permeating the whole Universe) and spacetime curvature arises naturally within the framework of field theory in curved spacetime (Birrell \& Davies 1982). The Lagrangian density for an interacting scalar field in a generic spacetime characterized by the metric tensor $g^{\mu \nu}$ is written as (Chernikov \& Tagirov 1968):

$$
\mathcal{L}=\sqrt{-g}\left[\frac{1}{2} g^{\mu \nu} \partial_{\mu} \phi \partial_{\nu} \phi-\frac{1}{2}\left(\mu^{2}+\xi R\right) \phi^{2}-\frac{\lambda}{4} \phi^{4}\right],
$$

where $\mu$ and $\lambda$ are the mass parameter and the self-coupling quartic coefficient of the Higgs field, respectively. In Eq. 1 we have also introduced the determinant of the metric $g^{\mu \nu}$ as $g$, and $\xi$, the coupling constant between the Higgs field $\phi$ and the Ricci scalar $R$. The coupling constant $\xi$ is a free parameter in any model so far imagined to describe scenarios of scalar fields coupled to gravity, and it is therefore important to extract this coefficient, or upper bounds, from phenomenological analyses.

The Higgs field develops, under spontaneous symmetry breaking, a vacuum expectation value $v_{0}=\left(-\mu^{2} / \lambda\right)^{1 / 2}$ in flat spacetime, and the masses of the fundamental fermions are proportional to $v_{0}$ via the Yukawa coefficients of the fermion-Higgs Lagrangian density term, $m_{i}=y_{i} v_{0} / \sqrt{2}$. The effective mass parameter of the Higgs field gets an extra-term due to the scalar curvature as $\mu^{2} \mapsto \mu^{2}+\xi R$, and the vacuum expectation value of the Higgs field will become spacetime dependent through the curvature scalar as:

$$
v=\sqrt{-\frac{\mu^{2}+\xi R}{\lambda}} \simeq v_{0}\left(1+\frac{\xi R}{2 \mu^{2}}\right),
$$

where the approximation holds in a weak-curvature limit. This implies that the mass $m_{i}$ of fundamental fermions, such as the electron, will be simply changed proportionally to the 
Higgs vacuum expectation value

$$
\delta m_{i}=\frac{y_{i}}{\sqrt{2}}\left(v-v_{0}\right) \simeq \frac{y_{i} \xi R v_{0}}{2^{3 / 2} \mu^{2}}=\frac{\xi R}{2 \mu^{2}} m_{i} .
$$

In other words, the presence of coupling of the Higgs field to space-time curvature adds to its inertial mass a contribution, which acts as a 'mass renormalization' due to curved spacetime (however, for an opposite interpretation of this mass shift see Faraoni \& Cooperstock (1998)). This mass shift is not present for protons and neutrons, due to the fact that their mass is primarily due to the gluonic fields which, being massless, are unaffected by the Higgs field. As discussed in more detail in Onofrio (2010), this implies that all molecular transitions only depending on the nuclei mass, such as vibrational and rotational spectra, should be unaffected by the Higgs-curvature coupling at leading order.

Unfortunately, the Ricci scalar outside spherically symmetric masses is zero, so we cannot use this coupling to infer possible mass shifts for the electrons. The only non-zero curvature scalar outside spherically symmetric masses is the Kreschmann invariant, and we will assume in the following considerations bounds to the Kreschmann coupling $\xi_{K}$ to the Higgs field in a Lagrangian of the form

$$
\mathcal{L}=\sqrt{-g}\left[\frac{1}{2} g^{\mu \nu} \partial_{\mu} \phi \partial_{\nu} \phi-\frac{1}{2}\left(\mu^{2}+\xi_{K} \Lambda_{\mathrm{Pl}}^{2} K\right) \phi^{2}-\frac{\lambda}{4} \phi^{4}\right],
$$

where $\Lambda_{\mathrm{Pl}}=\left(G \hbar / c^{3}\right)^{1 / 2}$ is the Planck length, whose value is $\Lambda_{\mathrm{Pl}} \simeq 10^{-35} \mathrm{~m}$ in conventional quantum gravity models, or larger values such as the one corresponding to models with early unifications of gravity to the other fundamental interactions (Arkani-Hamed et al. 1998, 1999). In the latter case the Planck length occurs at the $\mathrm{TeV}$ scale via extra-dimensions, $\Lambda_{\mathrm{Pl}} \simeq 10^{-19} \mathrm{~m}$, and in the following we will consider both these extreme situations.

Analogously to the case of the Ricci scalar, the mass parameter in the Higgs term then gets normalized as $\mu^{2} \mapsto \mu^{2}\left(1+\xi_{K} \Lambda_{\mathrm{Pl}}^{2} \lambda_{\mu}^{2} K\right)$, where we have introduced the Compton wavelength corresponding to the Higgs mass, $\lambda_{\mu}=\hbar /(\mu c)$, equal to $\lambda_{\mu} \simeq 1.6 \times 10^{-18} \mathrm{~m}$ if we assume $\mu=125 \mathrm{GeV}$. Notice that, due to the subattometer scale values of $\lambda_{\mu}$ and $\Lambda_{\mathrm{Pl}}$, an extremely large value of $\xi_{K}$ is necessary for having mass shifts of order unity or lower to compensate for Kreschmann invariants due to macroscopic curvature of any spacetime of astrophysical interest. In fact, we get a relative mass shift, for instance in the case of the electron, equal to

$$
\frac{\delta m_{e}}{m_{e}} \simeq \frac{1}{2} \xi_{K} \Lambda_{\mathrm{Pl}}^{2} \lambda_{\mu}^{2} K
$$

In the case of the Schwarzschild metric the Kretschmann invariant is $K=12 R_{s}^{2} / r^{6}$, with $R_{s}$ the Schwarzschild radius $R_{s}=2 G M / c^{2}$, and $r$ the distance from the center of the 
mass $M$. As a benchmark, for a solar mass white dwarf, $M=1 M_{\odot}$ with a Earth radius $R=R_{E} \simeq 9.1 \times 10^{-3} R_{\odot}$, we get $R_{s} \simeq 3 \times 10^{3} \mathrm{~m}$ and $K \simeq 2 \times 10^{-33} \mathrm{~m}^{-4}$. With $\Lambda_{\mathrm{Pl}} \simeq 10^{-35}$ $\mathrm{m}$ and the abovementioned value of $\lambda_{\mu}$ we obtain $\delta m_{e} / m_{e} \simeq 2.5 \times 10^{-139} \xi_{K}$ in MKSA units. For atomic transitions due to relocations of the electron in states with different principal quantum number, we expect that the mass shift affects the spectroscopy with a scaling of the transition wavelengths as $\delta \lambda / \lambda \simeq \delta m_{e} / m_{e}$, and therefore any evidence for a wavelength shift in the electronic transitions not accompanied by the same shift for transitions determined by the mass of the nuclei may be a distintive signature of Higgs-shifts. Therefore we need to detect emission or absorption wavelengths of both electronic and nuclear nature from a strong-gravity source, and make a comparison with either laboratory spectra or spectra gathered from weak-gravity astrophysical sources.

The spectrum of the $\mathrm{C}_{2}$ molecule has been the subject of extensive experimental and theoretical studies in molecular spectroscopy, and has been found in various astrophysical contexts including white dwarfs. In the visible region, the most prominent features of the $\mathrm{C}_{2}$ spectrum are the Swan bands, involving vibronic transitions between the electronic states $\mathrm{d}^{3} \Pi_{g}-\mathrm{a}^{3} \Pi_{u}$ (Huber \& Herzberg 1979; Brooke et al. 2013). For these transitions, in the presence of a Higgs-shift the electronic energy levels, proportional to the electron mass, should be shifted, while the vibrational levels, proportional to the nucleon mass, should stay constant. We therefore expect that the separation between different terms of the same Swan band should stay constant, the only effect of the Higgs shift being an overall shift of all the wavelengths. In the following we therefore focus on these specific spectra as gathered from two white dwarfs.

\section{Observations and data analysis on BPM 27606}

In the Bruce Proper Motion Survey, Luyten (1963) found BPM 276061, which has a dMe common proper motion companion. Eggen (1969) indicated that it is a white dwarf from $U B V$ photometry. Wegner (1973) discovered the strong $\mathrm{C}_{2}$ Swan bands which establish its spectral class as DQ in the current classification scheme, and discussed its kinematical properties (Wegner 1981). More detailed descriptions of the spectrum were given by Wickramasinghe \& Bessel (1979) and Wegner (1984). Atmospheric analyses (Koester et al. 1982; Wegner \& Yackovich 1984) showed that BPM 27606 has a helium dominated atmosphere $\left(\mathrm{C}: \mathrm{He} \sim 10^{-5}\right)$ and effective temperature $T_{\text {eff }} \sim 7,300 \mathrm{~K}$ and more recent studies of DQ atmospheres (e.g. Dufour et al. (2005)) leave this essentially unchanged.

\footnotetext{
${ }^{1}$ Other designations include: WD2154-512, GJ841B, L283-7, LDS765, LTT8768.
} 
In recent years many new DQ white dwarfs have been found (e.g. Kleinman et al. (2004)) and several new properties about them have been discovered. These include rapid variability of some of the hot ones (Williams et al. 2013), rotation (Lawrie et al. 2013), and strong magnetic fields (Williams et al. 2013). An additional problem that remains unsolved is the physics of the blueshifts in the Swan bands of the DQs. These were already measured in the milder cases of BPM 27606 and L 879-14 (Wegner 1984), but for cooler DQs this becomes more extreme, such as for LHS1126 (Bergeron et al. 1994). Hall \& Maxwell (2008) and Kowalski (2010) have reviewed possible mechanisms, but this subject is clearly hampered by the lack of information on the behaviour of the spectra of $\mathrm{CI}$ and $\mathrm{C}_{2}$ under white dwarf conditions.

BPM 27606 is a particularly good star for studying these effects because it is in the common proper motion system with CD- $51^{0} 13128$. This allows its true velocity to be known within fairly restrictive limits and the pair has a known distance from the trigonometric parallax. In addition, the shifts in the lines are relatively small $(\sim 4 \AA)$ and its magnetic field is not large. Vornanen et al. (2010) have reported a magnetic field measurement of $1.3 \pm 0.5 \mathrm{MG}$ from circular polarization of the $\mathrm{CH}$ bands near $\lambda 4300$. However the magnetic field may not be this high. From our new spectra this seems inconsistent with the lack of splitting of the $\lambda 4771 \mathrm{CI}$ line and $\mathrm{H} \alpha$, which indicates a magnetic field $B \leq 2 \times 10^{5} \mathrm{G}$. Although variability is possible, the line is single on all of our spectra as it was in the 1978 spectra of Wegner (1984).

The observations were made with the Robert Stobie Spectrograph (RSS) attached to the Southern African Large Telescope (SALT), which is described by Buckley et al. (2006). The RSS (Burgh et al. 2003; Kobulnicky et al. 2003)) employs Volume Space Holographic transmission gratings (VPHGs) and three E2V44-82 $2048 \times 4096$ CCDs with $15 \mu \mathrm{m}$ pixels separated by gaps of $1.5 \mathrm{~mm}$ width, or about $10-15 \AA$ at the dispersions used here.

All of the observations of BPM 27606 were obtained using a $1.0 \operatorname{arcsec} \times 8 \operatorname{arcmin}$ slit rotated by $71^{0}$ so that both the white dwarf and its bright companion could be observed simultaneously. A $2 \times 2$ pixel binning with a gain of $1.0 \mathrm{e}^{-} / \mathrm{ADU}$ gives a readout noise of $3.3 \mathrm{e}^{-} /$pixel. The $\mathrm{H}_{\alpha}$ region $(\lambda \lambda 6085-6925)$ which also covers the $C_{2}(0,2)$ bandhead was observed 2013 May 2 with the pg2300 grating and a pc04600 filter. Three 900 seconds exposures were obtained along with Ar comparison spectra. The FWHM measured from comparison lines is $1.3 \AA$. An identical set of exposures were secured 2014 May 12 to improve the signalto-noise ratio. The $\lambda \lambda$ 4700-5380 wavelength region which covers the $5135 \AA$ bandhead was observed 2013 May 19 (two exposures) and 2013 May 21 (four exposures). All exposure times were 812 seconds with the pg3000 grating, no filter, and CuAr comparisons were used, giving a FWHM of comparison lines of $1.0 \AA$. The $4737 \AA$ and $4382 \AA$ bandheads $(\lambda \lambda$ 
4326-5004) were observed 2013 May 5 and three 1042 second exposures using the pg3000 grating and no filter with a $\mathrm{CuAr}$ wavelength comparison. The resulting comparison line has a $\mathrm{FWHM}=1.1 \AA$. In 2014, two additional spectral regions were observed. Three 900 seconds exposures were secured 2014 May 13 of $\lambda \lambda 3540-4323$ with the pg3000 grating, no filter and a ThAr comparison producing a FWHM resolution of $1.3 \AA$ from companion lines. Three 663 seconds exposures covering $\lambda \lambda 5050-6010$ were made 2014 May 22 using the pg2300 grating with an Ar comparison giving a FWHM of $1.7 \AA$ for the comparison lines.

Starting from the bias subtracted and flattened images provided by the SALT pipeline (Crawford et al. 2010), the spectra were wavelength calibrated using the LONGSLIT menu in IRAF2. Fifth order polynomials were used for the wavelength calibrations in two dimensions, background subtraction, and the spectra were extracted using APSUM. Special care was taken to use portions of the frames adjacent to the spectra, to minimize the effects of the curved lines in the RSS. The final individual spectra were summed using IMCOMBINE and the CCDSUM option to remove cosmic rays. Examples of the resulting spectra are shown in Figures 1-5. The heliocentric velocity of the M2Ve companion, measured from our spectra using the $\mathrm{H}_{\alpha, \beta, \gamma}$ emission lines, was $-9.0 \pm 5.1 \mathrm{~km} / \mathrm{s}$, which we adopt here. This can be compared with $-9 \pm 1 \mathrm{~km} / \mathrm{s}$ (Wegner 1981) and $-8.1 \pm 1.7 \mathrm{~km} / \mathrm{s}$ (Karatas et al. 2004).

The observed wavelengths of the major bandheads of $\mathrm{C}_{2}$ are shown in Table 1 where they are compared with the wavelengths given by Pearce \& Gaydon (1976). These wavelengths refer to the minimum intensity at each bandhead from our spectra, and have been corrected to heliocentric values. We have analyzed the distance between two consecutive lines corresponding to the same $\Delta v=v^{\prime}-v^{\prime \prime}$, for the observed wavelengths $\Delta \lambda_{\text {obs }}(\Delta v)=\lambda_{\text {obs }}\left(v^{\prime}\right)-\lambda_{\text {obs }}\left(v^{\prime \prime}\right)$ and for the laboratory measured wavelengths $\Delta \lambda_{\text {lab }}(\Delta v)=\lambda_{\text {lab }}\left(v^{\prime}\right)-\lambda_{\text {lab }}\left(v^{\prime \prime}\right)$, evaluated both the overall average separation as $\langle\delta \lambda\rangle=N^{-1} \sum\left[\Delta \lambda_{\text {obs }}(\Delta v)-\Delta \lambda_{\text {lab }}(\Delta v)\right]$ and its dispersion as $\delta \lambda^{2}=N^{-1} \sum\left[\Delta \lambda_{\text {obs }}(\Delta v)-\Delta \lambda_{\text {lab }}(\Delta v)\right]^{2}$, obtaining the values of $\langle\delta \lambda\rangle=-0.71 \AA$ and $\left(\delta \lambda^{2}\right)^{1 / 2}=3.39 \AA$. The fact that $\langle\delta \lambda\rangle \ll\left(\delta \lambda^{2}\right)^{1 / 2}$ shows that, within the instrumental error, the spacing of the vibrational transitions is the same for the laboratory and the observed wavelengths. This provides a reliable anchor to study possible shifts in the bandheads due to the electron mass shift induced by the Higgs field. The stability of the vibrational transitions is further assured by their insensitivity to pressure-induced shifts since these are expected to be the same, in a linear approximation suitable for low pressures, for different vibronic bands (Lin 1973).

\footnotetext{
${ }^{2}$ IRAF is distributed by the National Optical Astronomy Observatories which are operated by the Association of Universities for Research in Astronomy, Inc. under cooperative agreement with the National Science Foundation.
} 


\subsection{Assessment of temperature and surface gravity}

Due to the lack of recent detailed spectral scans of BPM 27606, its effective temperature is estimated from photometry. Koester et al. (1982) used intermediate band Strömgren uby data from Wegner (1981) and $\log g=8$ models which yield an effective temperature $\mathrm{T}_{\text {eff }} \sim 7,600 \mathrm{~K}$ and $\log (\mathrm{He} / \mathrm{C}) \sim 4$.6. Using models of Galdikas (1985) these colours give $\mathrm{T}_{\text {eff }} \sim 7,200$ and $\log (\mathrm{He} / \mathrm{C}) \sim 4.9$. Giammichele et al. (2012) found $\mathrm{T}_{\text {eff }}=(7,193 \pm 92) \mathrm{K}$, $M=(0.60 \pm 0.07) M_{\odot}$, and $\log g=(8.03 \pm 0.04)$ in line, within few standard deviations, with our findings. Broadband UBV photometry, 2MASS near infrared colours (Carollo et al. 2003) and an atmosphere model by Wegner (1984) bracket these values, so for the present estimates we adopt $\mathrm{T}_{\text {eff }}=7,500 \mathrm{~K}$ and $\log (\mathrm{He} / \mathrm{C}) \sim 4.75$.

To estimate the mass, we first determine the white dwarf radius using the relationship $\log R / R_{0}=0.2\left(V_{0}-V\right)-\log \pi+4.914$ with $V_{0}=-25.60$ from Koester et al. (1982). The

visual magnitude $V=14.71$ is the mean of Eggen (1969) and Bergeron et al. (1994). For the parallax we adopt $\pi=0.068 \pm 0.003$, the mean of three parallax measurements: from Hipparcos (van Leeuwen 2007), the Yale parallax catalogue (van Altena et al. 1998), and the photometric parallax using the colour magnitude diagrams of Reid (http://www.stsci.edu $\tilde{\mathrm{inr}}$ /cmd.html) for the $\mathrm{dM}$ companion. This gives $R=0.0105 R_{\odot}$ which implies a mass $M \simeq 0.78 M_{\odot}$ according to the Hamada and Salpeter carbon or Chandrashekhar $\mu_{e}=2$ massradius relations. These values give a surface gravity of $\log g=8.3$ or $g=1.9 \times 10^{8} \mathrm{~cm} / \mathrm{s}^{2}$. The corresponding gravitational redshift would be $V_{R S}=0.635\left(M / M_{\odot}\right) /\left(R / R_{\odot}\right)=+47 \mathrm{~km} / \mathrm{s}$.

\subsection{Systematic effects}

The leading source of systematic effects in the $\mathrm{C}_{2}$ bands is pressure shifts in the dominant He background atmosphere, and in this section we estimate their order of magnitude. We interpolate in the DQ models of Galdikas (1984, 1985) for the atmospheric parameters at Rosseland mean optical depth $\bar{\tau}=0.1$ for $\mathrm{T}_{\text {eff }}=7,500 \mathrm{~K}$ and $\log (\mathrm{C} / \mathrm{He})=-4.75$ for the order of magnitude of conditions in the line forming region. This is $T_{0.1}=6,500 \mathrm{~K}$ and $P_{g}=1.8 \times 10^{9}$ dynes $\mathrm{cm}^{-3}$. As the models use $g=10^{8} \mathrm{~cm} \mathrm{~s}^{-2}$, and for BPM 27606 $g=1.9 \times 10^{8} \mathrm{~cm} \mathrm{~s}^{-2}$, the number density is scaled to be $3.8 \times 10^{21} \mathrm{~cm}^{-3}$.

Hammond (1990) measured pressure shifts for the five $C_{2}$ bandheads observed here in He under conditions close to those in BPM $27606\left(T=4,200 \mathrm{~K}\right.$ and $\left.N=3 \times 10^{21} \mathrm{~cm}^{-3}\right)$. Although these measurements cannot explain the large blueshifts $\Delta \lambda \sim-200 \AA$ in the cooler peculiar DQ stars (Kowalski 2010; Hall \& Maxwell 2008), the shifts in BPM 27606 are much smaller than this and the laboratory measurements are of similar size, so it seems reasonable 
that for this star they can be used to estimate the pressure shifts. If the line broadening in the Swan bands resembles van der Waals broadening, the pressure shifts measured by Hammond (1990), $\Delta \lambda_{\text {Ham }}$, would scale as

$$
\Delta \lambda_{\text {press }}=\left(\frac{T_{0.1}}{4,200}\right)^{0.3}\left(\frac{N_{0.1}}{3 \times 10^{21}}\right) \Delta \lambda_{\text {Ham }},
$$

with $T_{0.1}$ expressed in $\mathrm{K}$ and $N_{0.1}$ in $\mathrm{cm}^{-3}$. For the estimated conditions at $\bar{\tau}=0.1, \Delta \lambda_{\text {press }}=$ $1.44 \Delta \lambda_{\text {Ham }}$, which is used to correct the measured wavelengths of the $C_{2}$ bandheads given in Table 2 as $\Delta \lambda_{\text {fin }}=\Delta \lambda_{\text {meas }}-\Delta \lambda_{\text {press }}-\Delta \lambda_{\mathrm{GR}}$, where $\Delta \lambda_{\mathrm{GR}}$ is the gravitational redshift. The major source of systematic error in Equation 6 is the choice of $\bar{\tau}=0.1$ for the model atmosphere. This turns out to be relatively insensitive, as increasing the Rosseland optical depth to $\bar{\tau}=0.2$ would multiply $\Delta \lambda_{\text {press }}$ in Tables 2 and 3 by a factor 1.2 .

We also detect a weak feature at $\lambda=6563.33 \AA$. If this is the $H_{\alpha}$ line, $\lambda=6562.82$ $\AA$, no pressure shifts are expected, and this gives a gravitational redshift $V_{R S}=+52 \mathrm{~km} / \mathrm{s}$. We do not detect the $H_{\beta}$ line in our spectra. The $\mathrm{CH}(0,0)$ band at $4314.2 \AA$ appears to be present as a weak feature at $\lambda 4299$ and helps to confirm the presence of hydrogen.

A weak CI line can be seen in Figure 2 (see also Fig. 3 for details). The measured heliocentric wavelength neglecting pressure shifts is $4771.1 \AA$. The laboratory wavelength of the strongest component of the $3 s^{1} P^{0}-4 p^{3} P$ multiplet is $4771.75 \AA$ (Moore 1993). There are currently no data on pressure shifts of CI lines. The magnetic field on the surface of BPM 27606 is estimated looking at the resolution of the CI line. The classical Zeeman effect yields the relationship $\Delta \lambda=4.7 \times 10^{-5} \lambda^{2} B$ (in cgs units). The fact that only a single line with FWHM $\simeq \Delta \lambda=2.0 \AA$ is visible (although with a low signal-to-noise ratio), yields an upper bound on the magnetic field of $B \leq 2 \times 10^{5} \mathrm{G}$, which makes negligible any correction to the estimates above. Using detailed calculations by Williams et al. (2013) of the magnetic splitting of this line also suggest such a low magnetic field.

The orbital motion is estimated considering the presence of the common proper motion companion to BPM 27606 with $V=10.49$, of spectral type M2Ve $\left(M=0.4 M_{\odot}\right)$ at a projected separation of 28.45 arcsec, which at 15 pc yields a separation of $4.1 \times 10^{2} \mathrm{AU}$. With a white dwarf mass of $0.8 M_{\odot}$ and taking this to be the semimajor axis of a circular orbit, the orbital period would be $P \sim 7.6 \times 10^{3}$ years and the projected orbital velocity would be $\pm 0.5 \mathrm{~km} / \mathrm{s}$, which is the order of magnitude of an additional source of uncertainty in the gravitational redshift determination.

For BPM 27606 we conclude that an upper bound to the wavelength difference between the molecular $\mathrm{C}_{2}$ bands and the $\mathrm{H}_{\alpha}$ and $\mathrm{CI}$ atomic lines is $1.3 \AA$. This is obtained by summing up the variance of the estimated pressure shifts (the square of the error in the 
second column of Table 2, corrected by the 1.44 Hammond scaling factor) and the square of the final difference between the processed and the laboratory wavelength (last column in Table 2), all other systematic errors being negligible with respect to these sources. If we disqualify the troubling CI line, it is $0.7 \AA$. Although the corrections for pressure shifts in the $\mathrm{C}_{2}$ bands seems satisfactory, one must consider the uncertainties in their measurements which dominate the error budget in our estimate of the difference and is of the order of \pm 2 $\AA$.

\section{Observations and data analysis on Procyon B}

Procyon B is another white dwarf that has both atomic and molecular features in its spectrum and is in a well known binary. Its orbit and mass have been long studied (e.g. Schaeberle (1896); Spencer Jones (1928); Strand (1951)). Girard et al. (2000) obtained masses of $m_{A}=1.495 M_{\odot}$ and $m_{B}=0.602 M_{\odot}$ for the two components. Although Procyon B was long known to be a white dwarf, its spectrum could not be studied due to its proximity to its bright $(V=+0.34)$ primary (separation $\leq 5$ arcsec). Provencal et al. (2002) secured spectra using the STIS instrument on the HST in 1998 February (Proposal 7398; PI H.L. Shipman) where the observations and data reductions are detailed. Here we adopt the atmospheric analysis in Provencal et al. (2002) which gives $\mathrm{T}_{\text {eff }}=(7,740 \pm 150) \mathrm{K}, R / R_{\odot}=$ $0.0124 \pm 0.00032$ and $\log C / H e=-5.5$. These parameters show that Procyon B lies close to the carbon white dwarf mass-radius relation and predict a surface gravity of $g=1.1 \times$ $10^{8} \mathrm{~cm} \mathrm{~s}^{-2}$ and a gravitational redshift of $V_{R S}=+31 \mathrm{~km} / \mathrm{s}$.

From the HST archives we used images 04g802010, 04g802020, 04g8020j0, and 04g802090 which have suitable signal to noise ratio. We measured the wavelengths for $C_{2}$ bands, $\mathrm{MgII}$ and CaII lines. Figure 6 shows the $C_{2}$ bands near $4.737 \AA$ and Figure 7 shows the CaII lines. The remainder of the spectrum is shown in Provencal et al. (2002). The orbital velocity from Irwin (1992) for the radial motion of Procyon B in 1998 is $-8.8 \mathrm{~km} / \mathrm{s}$. The models of Galdikas (1985) using the $\mathrm{T}_{\text {eff }}$ and $g$ above indicate at $\bar{\tau}=0.1, \mathrm{~T}_{0.1}=6,750 \mathrm{~K}$, and $\mathrm{N}_{0.1}=3.5 \times 10^{21} \mathrm{~cm}^{-3}$.

The $C_{2}$ pressure shifts of Hammond (1990) discussed in Section 3.2 are thus multiplied by 1.3. The pressure shifts of the observed CaII and $\mathrm{Mg}$ lines produced by He are available and in both cases are redshifts. For CaII, theoretical values (Monteiro et al. 1986) and laboratory measurements (Hammond 1989) agree relatively well. Monteiro et al. (1986) calculated pressure shifts for MgII and these were scaled to the above atmospheric parameters assuming van der Waals broadening. Table 3 summarizes the laboratory and measured wavelengths of features in the spectrum of Procyon B along with the corrections due to the 
orbital motion, $\Delta \lambda_{\text {orbit }}$, the estimated pressure shifts $\Delta \lambda_{\text {press }}$, and the gravitational redshift, $\Delta \lambda_{\mathrm{GR}}$. The resulting corrected wavelength of each line, $\lambda_{\text {corr }}$, and the residual $\Delta \lambda_{\text {fin }}=$ $\lambda_{\text {lab }}-\lambda_{\text {corr }}$ are given. These are consistent with the results for BPM 27606. By repeating the analysis as for the latter, and using the Hammond scaling factor of 1.3, the difference between atomic and molecular lines is formally $0.5 \AA$, with the same uncertainties due to pressure shifts as before.

\section{Upper bounds to the Higgs-curvature coupling}

Based on the absence of relative shifts between the electronic and the vibrational transitions of the Swan bands, we are able to assess upper bounds on the Higgs-Kreschmann coupling. The expected wavelength shift is

$$
\frac{\delta \lambda}{\lambda} \simeq \frac{1}{2} \xi_{K} \Lambda_{\mathrm{Pl}}^{2} \lambda_{\mu}^{2} K
$$

where the Kreschmann invariant is, for the estimated values of the mass and radius of BPM 27606, $K=12 R_{s}^{2} / R^{6} \simeq 4.2 \times 10^{-34} \mathrm{~m}^{-4}$. By assuming $\Lambda_{\mathrm{Pl}}=10^{-35} \mathrm{~m}$, and the value of $\lambda_{\mu}$ quoted in Section 2 , we obtain $\delta \lambda / \lambda \simeq 5.4 \times 10^{-140} \xi_{K}$, which may be inverted yielding an upper bound, for an average wavelength of $\lambda=5,000 \AA$

$$
\xi_{K} \leq 3.6 \times 10^{135}\left(\frac{\delta \lambda_{\text {est }}}{1 \AA}\right),
$$

where $\delta \lambda_{\text {est }}$ (in $\AA$ ) is the estimated wavelength resolution. If instead we use $\Lambda_{\mathrm{Pl}}=10^{-19} \mathrm{~m}$ as in models with extra dimension and quantum gravity at the Fermi scale (Arkani-Hamed et al. 1998, 1999), the bounds are a bit more constraining, as $\xi_{K} \leq 3.6 \times 10^{103}$ at $\delta \lambda_{\text {est }}=1 \AA$.

Both examples of upper bounds are expressed in meter-kilogram-second-ampere (MKSA) units of the Systeme International (SI), and it is worth converting them into natural units for the benefit of a comparison to the bounds already estimated from the observation of the Higgs particle at LHC (Atkins \& Calmet 2013; Xianyu et al. 2013). The action term for the Higgs field including its coupling to spacetime, taking into account explicitly $\hbar$ and $c$, is expressed in SI units in terms of, for instance, the mass term as $S \propto \int d^{3} x d(c t) \frac{m^{2} c^{2}}{\hbar^{2}} \phi^{2}$. Then the scalar field has dimensions $[\phi]=M^{1 / 2} T^{-1 / 2}$, and the Higgs-curvature term satisfying $S \propto \int d^{3} x d(c t) \xi_{S I} \phi^{2} R$ implies that $\xi$ is dimensionless. If the analysis is repeated for the action term expressed in natural units (NU), with $\hbar=1, c=1$, the dimensions of the scalar field change accordingly, and from the mass term it is simple to infer that $\phi_{N U}^{2}=(c / \hbar)^{2} \phi^{2}$, implying the following relationship between the $\xi$ parameter evaluated in the two units sys- 
tems, $\xi_{N U}=(\hbar / c)^{2} \xi_{S I}=1.23 \times 10^{-85} \xi_{S I}$. Analogous considerations may be repeated for the Higgs-Kreschmann coupling in which the curvature term has the same dimensions since $R$ is replaced by $\Lambda_{\mathrm{Pl}}^{2} K$. This implies that in natural units the bounds on the Higgs-Kreschmann coupling become respectively, for the two extreme choices of $\Lambda_{\mathrm{Pl}}$, $\xi_{K} \leq 4.4 \times 10^{50}$ (for $\Lambda_{\mathrm{Pl}}=10^{-35} \mathrm{~m}$ ) and $\xi_{K} \leq 4.4 \times 10^{18}$ (for $\Lambda_{\mathrm{Pl}}=10^{-19} \mathrm{~m}$ ). This second bound on the HiggsKreschmann coupling is quantitatively comparable to the ones assessed on the Higgs-Ricci coupling through measurements at the LHC as reported in Atkins \& Calmet (2013) and Xianyu et al. (2013), although in their analyses the usual $\Lambda_{\mathrm{Pl}}=10^{-35} \mathrm{~m}$ is assumed. With respect to bounds from table-top experiments based on tests of the superposition principle for gravitational interactions as discussed in Onofrio (2012), the bounds derived in this paper represent an improvement by ten orders of magnitude, as shown in Table 4.

\section{Conclusions}

We have shown that observations in a somewhat controlled environment like the one provided by carbon-rich white dwarfs may be used to give upper bounds on the coupling between the Higgs field and a specific invariant of the curvature of the spacetime, such as the Kreschmann invariant. The existence of nonminimal couplings between the Higgs and spacetime curvature is crucial to various proposals in which the Higgs also plays the role of the inflaton (Bezrukov \& Shaposhnikov 2008; Bezrukov et al. 2009), and as a mechanism to suppress the dark energy contribution of quantum fields to the level compatible with the astrophysical observations based on SNIa (Shapiro \& Solà 2000).

This methodology is complementary to the upper bounds recently discussed arising from the LHC experiments and their degree of agreement with the standard model of elementary particle physics (Atkins \& Calmet 2013; Xianyu et al. 2013), and can be adopted also to search for couplings between generic scalar fields, not necessarily directly related to the Higgs vacuum, and space-time curvature, which may be competitive with bounds arising from the analysis of the cosmic background radiation as reported in Hwang (1998); Komatsu \& Futamase (1998, 1999). Scalar fields, even if not directly interacting among themselves at level of their classical Lagrangian, will have a crosstalk once quantum radiative corrections are considered - the very origin of the hierarchy problem in Grand Unified Theories - so any scalar field will be then coupled with the Higgs field and will indirectly affect the mass of elementary particles. Bounds based on this analysis could be even more stringent as many of the proposed candidates, for instance scalar fields invoked to accommodate the acceleration of the Universe (Peebles \& Ratra 1988; Ratra \& Peebles 1988; Wetterich 1988; Ostriker \& Steinhardt 1995; Caldwell et al. 1998; Carroll 1998; Bahcall et al. 1999; 
Wang et al. 2000) have a Compton wavelength greatly exceeding the one associated to the Higgs field, provided that compact astrophysical sources with non-zero Ricci scalar may be found.

The spectroscopic analysis presented here could be improved in a number of ways in the near future. Measurements and calculations of the pressure shifts of $\mathrm{C}$ I lines and the

$C_{2}$ Swan bands under white dwarf conditions are needed. Observations of the ultraviolet carbon and other metal lines which are from lower energy levels would help disentangle the pressure shifts from the gravitational redshift measurement. A detailed scan of the white dwarf's spectral energy distribution combined with an updated atmosphere model would help understanding the details of the molecular and atomic carbon features. Additional objects of this type that are in binary pairs would help in the assessment of the local gravity, further diversifying the sample to counterbalance peculiar systematic effects.

We are grateful to Susanne Yelin for a critical reading of the manuscript. Some of the observations reported in this paper were obtained with the Southern African Large Telescope (SALT) under proposals 2013-1-DC-001 and 2014-1-DC-001. Additional data used observations made with the NASA/ESA Hubble Space Telescope, and were obtained from the Hubble Legacy Archive, which is a collaboration between the Space Telescope Science Institute (STScI/NASA), the Space Telescope European Coordinating Facility (STECF/ESA) and the Canadian Astronomy Data Centre (CADC/NRC/CSA). This work was also partially funded by the National Science Foundation through a grant for the Institute for Theoretical Atomic, Molecular, and Optical Physics at Harvard University, and the Smithsonian Astrophysical Laboratory.

\section{REFERENCES}

Aad, G., et al. (ATLAS Collaboration) 2012, Phys. Lett. B, 716, 1

Adams, W. S., 1925, Proc. Nat. Acad. Sci., 11, 382

Arkani-Hamed, N., Dimopoulos, S., \& Dvali, G. 1998, Phys. Lett. B, 429, 263; Phys. Lett. B, 436,257

Arkani-Hamed, N., Dimopoulos, S., \& Dvali, G. 1999, Phys. Rev. D, 59, 086004

Atkins, M., \& Calmet, X. 2013, Phys. Rev. Lett., 110, 051301

Bahcall, N.A., Ostriker, J.P., Permutter S., \& Steinhardt, P.J. 1999, Science, 284, 1481 
Berengut, J.C., et al. 2013, Phys. Rev. Lett., 111, 010801

Bergeron, P., Ruiz, M.-T., Leggett, S.K., Saumon, \& D., Wesemael, F. 1994, ApJ, 423, 456

Bezrukov, F., \& Shaposhnikov, M. 2008, Phys. Lett. B, 659, 703

Bezrukov, F., Magnin, A., \& Shaposhnikov, M. 2009, Phys. Lett. B, 675, 88

Birrell, N.D., \& Davies, P.C.W. 1982, Quantum Fields in Curved Space, (Cambridge University Press)

Brooke, J.S.A., Bernath, P.F., Schmidt, T.W., \& Bacskay, G.B. 2013, J. Quantit. Spectroscopy \& Radiative Transfer, 124, 11

Buckley, D.A., Swart, G.P., \& Meiring, J.G. 2006, SPIE, 6267, 32

Burgh, E.B., et al. 2003, SPIE, 4841, 1463

Caldwell, R.R., Dave, R., \& Steinhardt, P.J. 1998, Phys. Rev. Lett., 80, 1582

Carollo, D., Koester, D., Spagna, A., Lattanzi, M.G., \& Hodgkin, S.T. 2003, A \& A, 400, L13

Carroll, S.M. 1998, Phys. Rev. Lett., 81, 3067

Chandrasekhar, S. 1935, MNRAS, 95, 207

Chatrchiyan, S., et al. (CMS Collaboration) 2012, Phys. Lett. B, 716, 30

Chernikov, N. A., \& Tagirov, E. A. 1968, Ann. Inst. Henri Poincare, Sect. A 9, 109.

Crawford, S. M., et al. 2010, SPIE, 7737, 54

Deser, S., \& van Niewenhuizen, P. 1974, Phys. Rev. D, 10, 401

Dufour, P., Bergeron, P., \& Fontaine, G. 2005, ApJ, 627, 404

Eggen, O.J. 1969, ApJ, 157, 287

Faraoni, V., \& Cooperstock, F. I. 1988, Eur. J. Phys., 19, 419

Galdikas, A. 1984, Ap \& SS, 105,39

Galdikas, A. 1985, Vilniaus Astron. Observ. Biulet. 73, 24

Giammichele, N., Bergeron, P., \& Dufour, P. 2012, ApJ Suppl. Ser., 199, 1 
Girard, T.M. et al. 2000, AJ, 119, 2428

Hall, P.B., \& Maxwell, A.J. 2008, ApJ, 678, 1292

Hammond, G.L. 1989, in White Dwarfs, Lecture Notes in Physics, Vol. 328, G. Wegner, ed. (Berlin: Springer-Verlag), p. 346

Hammond, G.L. 1990, AIPC, 216, 611

Hehl F.W., Ne'eman, Y., Nitsch, J., \& von der Heyde, P. 1978, Phys. Lett. B, 78, 102

Huber, K., \& Herzberg, G. 1979, Molecular spectra and molecular structure, Volume IV: constants of diatomic molecules, (Van Nostrand Reinhold)

Hut, P. 1981, Phys. Lett., 99B, 174

Hwang, J., \& Noh, H. 1998, Phys. Rev. Lett., 81, 5274

Irwin, A.W., Fletcher, J.M., Yang, S.L.S., \& Walker, G.A.H., Goodenough, C. 1992, P.A.S.P 104,489

Karatas, Y., Bilin, S., Eker, Z., \& Demircano, O. 2004, MNRAS, 349, 1069

Kleinman, S.J., et al. 2004, ApJ, 607, 426

Kobulnicky, H.A. et al. 2003, SPIE, 4841, 1634

Koester, D., Weidemann V., \& Zeidler, E.-M. 1982, A \& A, 116, 147

Komatsu, E., \& Futamase, T. 1998, Phys. Rev. D, 58, 023004

Komatsu, E., \& Futamase, T. 1999, Phys. Rev. D, 59, 064029

Kowalski, P.M. 2010, A\&A, 519, L8

Lawrie, K.A., Burleigh, M.R., Dufour, \& M.R., Hodgkin, S.T. 2013, MNRAS, 433, 1599

Lin, S.H. 1973, J. Chem. Phys., 59, 4458

Lovelock, D. 1971, J. Math. Phys., 12, 498

Luyten, W.J. 1963, Bruce proper motion surveys: The general catalogue, Vol. I, II

Monteiro, T., Cooper, I., Dickinson, A., \& Lewis, E. 1986, J. Phys. B, 19, 4087 
Moore, C. E. 1993, in Tables of Spectra of Hydrogen, Carbon, Nitrogen and Oxygen atoms and ions, CRC Series in Evaluated Data in Atomic Physics, J. M. Gallagher ed. (Boca Raton: CRC Press)

Onofrio, R. 2010, Phys. Rev. D, 82, 065008

Onofrio, R. 2012, Eur. Phys. J. C, 72, 2006

Ostriker, J.P., \& Steinhardt, P.J. 1995, Nature (London), 377, 600

Pearse, R.W.B., \& Gaydon, A.G. 1976, The Identification of Molecular Spectra, 4th Ed. (London: Chapman and Hall)

Peebles, P.J.E., \& Ratra, B. 1988, ApJ, 325, L17

Provencal, J.L., Shipman, H.L., Koester, D., Wesemael, F., \& Bergeron, P. G. 2002, ApJ, 568,324

Ratra B., \& Peebles, P.J.E. 1988, Phys. Rev. D, 37, 3406

Schaeberle, J.M. 1896, AJ, 17, 37

Shapiro, I.L., \& Solà, J. 2000, Phys. Lett. B, 475, 236

Sotiriou, T.P., \& Faraoni, V. 2010, Rev. Mod. Phys.,82, 451

Spencer Jones, H. 1928, MNRAS, 88, 403

Stelle, K.S. 1977, Phys. Rev. D, 16, 953

Strand, K.A., 1951, ApJ, 113, 1

van Altena, W.F., Lee, J.T., \& Hoffleit, E.D. 1995, Yale Trigonometric Parallaxes, Online data Vizier Service (4th ed.; New Haven, CT: Yale University Observatory)

van Leeuwen, F. 2007, A \& A, 474, 653

Vornanen, T., Berdyugina, S.V., Berdyugin, A.V., \& Piirola, V. 2010, ApJ, 720, L52

Wang. L.M., Caldwell R.R., \& Ostriker, J.P., \& Steinhardt, P.J. 2000, ApJ, 530, 17

Wegner, G. 1973, MNRAS, 163, 381

Wegner, G. 1981, AJ, 86, 264

Wegner, G. 1984, Ap\&SS, 104, 347 
Wegner., G., \& Yackovich, F.H. 1984, ApJ, 284, 257

Wegner., G. 1989, in White Dwarfs, Lecture Notes in Physics, Vol. 328, G. Wegner, ed. (Berlin: Springer-Verlag), p. 346

Wetterich., C. 1988, Nucl. Phys. B, 302, 668

Wickramasinghe, \& D.T., Bessel, M.S. 1979, MNRAS, 188, 841

Williams, K.A., et al. 2013, ApJ, 769, 123

Xianyu, Z.-Z., Ren J., \& He, H.-J. 2013, Phys. Rev. D, 88, 096013 
Table 1: Observed heliocentric wavelengths of the Swan bands from BPM 27606 for $v^{\prime}, v^{\prime \prime}$ transitions, and comparison to the laboratory wavelengths, all expressed in $\AA$ units.

\begin{tabular}{cccc}
\hline \hline$v^{\prime}, v^{\prime \prime}$ & $\lambda_{\text {obs }}$ & $\lambda_{\text {lab }}$ & $\lambda_{\text {obs }}-\lambda_{\text {lab }}$ \\
\hline 0,2 & 6179.90 & 6191.2 & -11.3 \\
1,3 & 6114.74 & 6122.1 & -7.4 \\
\hline 0,1 & 5630.35 & 5635.5 & -5.15 \\
1,2 & 5580.07 & 5585.5 & -5.4 \\
2,3 & 5536.00 & 5540.7 & -4.7 \\
3,4 & 5495.64 & 5501.9 & -6.3 \\
4,5 & 5467.73 & 5470.3 & -2.6 \\
\hline 0,0 & 5159.97 & 5165.2 & -5.2 \\
1,1 & 5125.64 & 5129.3 & -3.7 \\
2,2 & 5094.45 & 5097.7 & -3.3 \\
\hline 1,0 & 4733.05 & 4737.1 & -4.0 \\
2,1 & 4712.04 & 4715.2 & -3.2 \\
3,2 & 4694.74 & 4697.6 & -2.9 \\
4,3 & 4682.06 & 4684.8 & -2.7 \\
5,4 & 4675.53 & 4678.6 & -3.4 \\
6,5 & 4679.89 & 4680.2 & -0.3 \\
\hline 2,0 & 4378.86 & 4382.5 & -3.6 \\
3,1 & 4368.07 & 4371.4 & -3.3 \\
4,2 & 4362.24 & 4365.2 & -3.0 \\
\hline
\end{tabular}


Table 2: Data processing for the extraction of the wavelength shift on BPM 27606, with all wavelengths expressed in $\AA$ units.

\begin{tabular}{ccccccc}
\hline \hline line & $\Delta \lambda_{\text {Ham }}$ & $\Delta \lambda_{\text {press }}$ & $\Delta \lambda_{\text {meas }}$ & $-\Delta \lambda_{\text {comp }}$ & $\Delta \lambda_{\mathrm{GR}}$ & $\Delta \lambda_{\text {fin }}$ \\
$(1)$ & $(2)$ & $(3)$ & $(4)$ & $(5)$ & $(6)$ & $(7)$ \\
\hline $\mathrm{C}_{2}(0,2)$ & $-8.4 \pm 0.3$ & -12.1 & -11.3 & +0.18 & -1.0 & 0.0 \\
$\mathrm{C}_{2}(0,1)$ & $-3.2 \pm 0.5$ & -4.6 & -5.15 & +0.17 & -0.9 & -1.3 \\
$\mathrm{C}_{2}(0,0)$ & $-3.2 \pm 0.5$ & -4.6 & -5.2 & +0.16 & -0.8 & +0.0 \\
$\mathrm{C}_{2}(1,0)$ & $-3.8 \pm 1.0$ & -5.5 & -4.0 & +0.14 & -0.7 & +0.9 \\
$\mathrm{C}_{2}(2,0)$ & $-3.1 \pm 1.0$ & -4.5 & -3.6 & +0.13 & -0.7 & +0.3 \\
\hline $\mathrm{CI}(\lambda 4771)$ & - & - & -0.65 & +0.15 & -0.75 & -1.2 \\
$\mathrm{H}_{\alpha}$ & - & - & +0.5 & +0.20 & -1.0 & -0.3 \\
\hline
\end{tabular}

Note. - Column (1) is the name of the line. Column (2) are Hammond's (1990) pressure shifts measurement for the $\mathrm{C}_{2}$ bands and their errors. Column (3) are the estimated pressure shifts in BPM 27606 using (2) and scaled as described in Section 3.2. Column (4) is the difference between the measured heliocentric wavelength from SALT spectra and laboratory wavelengths. Column (5) is the correction for the system's motion based on the companion's radial velocity $V_{r}=-9.1 \mathrm{~km} / \mathrm{s}$. Column (6) is the correction for a gravitational redshift of $47 \mathrm{~km} / \mathrm{s}$. Column (7) is the final difference between the processed white dwarf and laboratory wavelengths. 
Table 3: Data processing for the extraction of the wavelength shift on Procyon B, with all wavelengths expressed in $\AA$ units.

\begin{tabular}{cccccccc}
\hline \hline line & $\lambda_{\text {lab }}$ & $\lambda_{\text {obs }}$ & $\Delta \lambda_{\text {orbit }}$ & $\Delta \lambda_{\text {press }}$ & $\Delta \lambda_{\text {GR }}$ & $\lambda_{\text {corr }}$ & $\Delta \lambda_{\text {fin }}$ \\
$(1)$ & $(2)$ & $(3)$ & $(4)$ & $(5)$ & $(6)$ & $(7)$ & $(8)$ \\
\hline $\mathrm{C}_{2}(0,0)$ & 5165.2 & 5161.8 & -0.15 & +4.2 & -0.53 & 5165.3 & +0.1 \\
$\mathrm{C}_{2}(1,1)$ & 5129.3 & 5126.5 & -0.15 & & & & \\
$\mathrm{C}_{2}(1,0)$ & 4737.1 & 4733.1 & -0.14 & +4.9 & -0.49 & 4737.4 & +0.3 \\
$\mathrm{C}_{2}(2,1)$ & 4715.2 & 4714.5 & -0.14 & & -0.49 & & \\
$\mathrm{C}_{2}(3,2)$ & 4697.6 & 4696.1 & -0.14 & & -0.48 & & \\
$\mathrm{CaII} \mathrm{H}$ & 3968.47 & 3969.84 & -0.12 & -1.4 & -0.41 & 3967.9 & -0.6 \\
$\mathrm{CaII} \mathrm{K}$ & 3933.66 & 3935.18 & -0.11 & -0.4 & -0.41 & 3934.3 & +0.7 \\
$\mathrm{MgII}$ & 2802.70 & 2803.10 & -0.08 & -0.5 & -0.29 & 2802.2 & -0.5 \\
$\mathrm{MgII}$ & 2795.53 & 2796.85 & -0.08 & -0.6 & -0.29 & 2795.9 & +0.4 \\
\hline
\end{tabular}

Note. - Column (1) is the name of the line. Column (2) is the laboratory wavelength. Column (3) is the measured heliocentric wavelength using the HST reduction. Column (4) is the wavelength correction due to the orbital motion of Procyon B. Column (5) gives pressure shift corrections described in Section 4. Column (6) is the correction for the gravitational redshift assuming that it is $+31 \mathrm{~km} / \mathrm{s}$. Column (7) is the corrected wavelength of the observed line. Column (8) is the final difference between Procyon B and laboratory wavelengths, $\Delta \lambda_{\text {fin }}=\lambda_{\text {corr }}-\lambda_{\text {lab }}$. 
Table 4: Summary of the upper bounds on Kretschmann-Higgs couplings $\xi_{K}$ (in natural units) from the analysis of the two white dwarfs (first two rows) and comparison to limits inferred from tabletop experiments on the validity of the superposition principle for gravitational interactions as discussed in Onofrio (2012) (last row). Upper bounds to the Higgs-Kreschmann coupling constant $\xi_{K}$ have been evaluated for two different values of the Planck length as in the last two columns, the standard one and one assuming unification of gravity with the gauge interactions at the Fermi scale, respectively. The better wavelength resolution estimated for Procyon B is partly offset by the smaller value of its mass and the larger value of its radius with respect to BPM 27606, which affect significantly the bound due to the strong dependence of the Kreschmann invariant on mass and radius.

\begin{tabular}{cccccc}
\hline \hline System & $R / R_{\odot}$ & $M / M_{\odot}$ & $\delta \lambda_{\text {est }}(\AA)$ & $\xi_{k}\left(\Lambda_{\mathrm{Pl}}=10^{-35} \mathrm{~m}\right)$ & $\xi_{k}\left(\Lambda_{\mathrm{Pl}}=10^{-19} \mathrm{~m}\right)$ \\
\hline BPM 27606 & 0.0105 & 0.78 & 1.3 & $5 \times 10^{50}$ & $5 \times 10^{18}$ \\
Procyon B & 0.0124 & 0.602 & 0.5 & $9 \times 10^{50}$ & $9 \times 10^{18}$ \\
Table-top experiments & & & & $2.5 \times 10^{60}$ & $2.5 \times 10^{28}$ \\
\hline
\end{tabular}




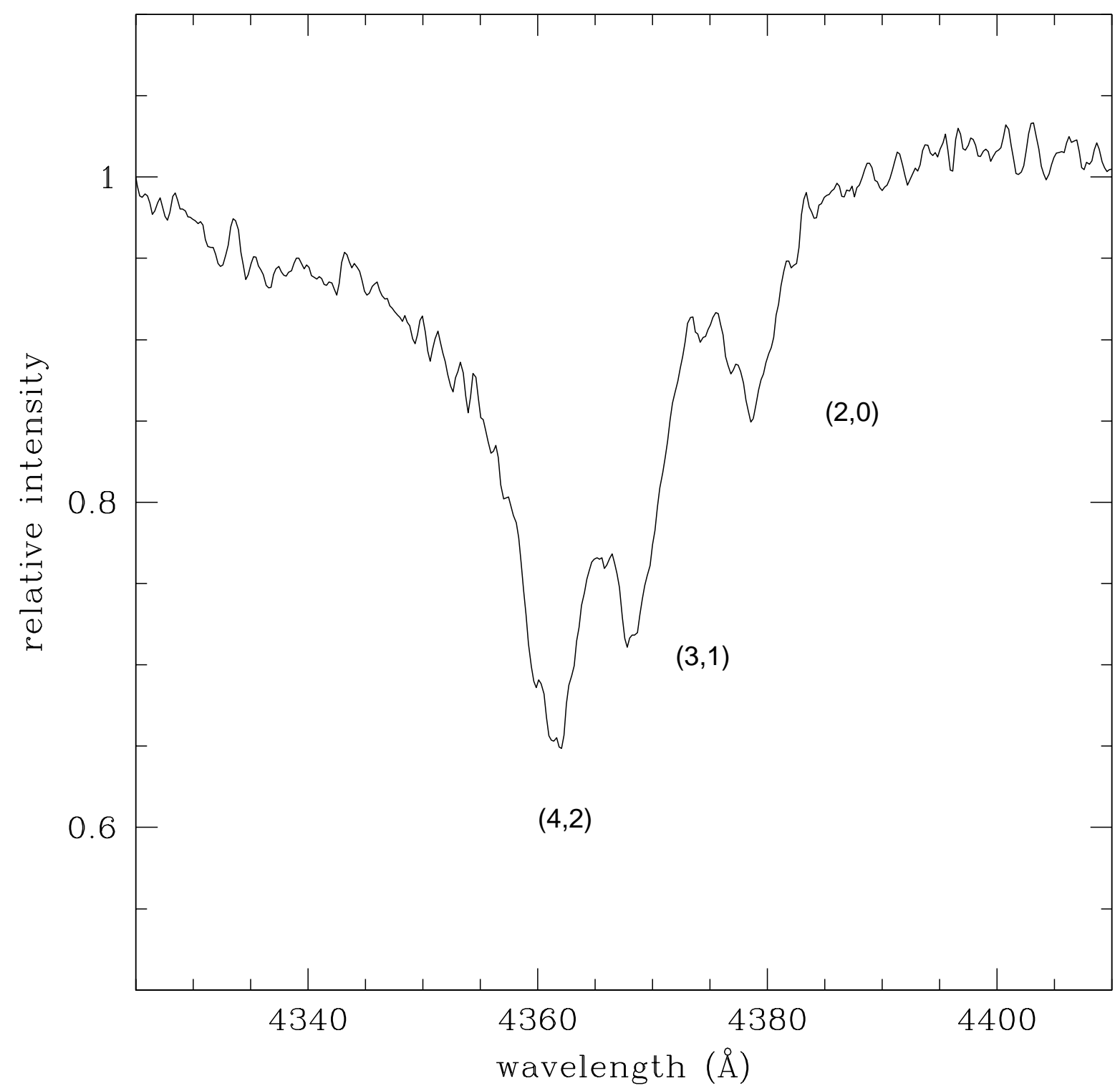

Fig. 1.- Portion of SALT spectra of BPM 27606 showing the Swan band with $\Delta v=2$ of $C_{2}$ near $4370 \AA$. 


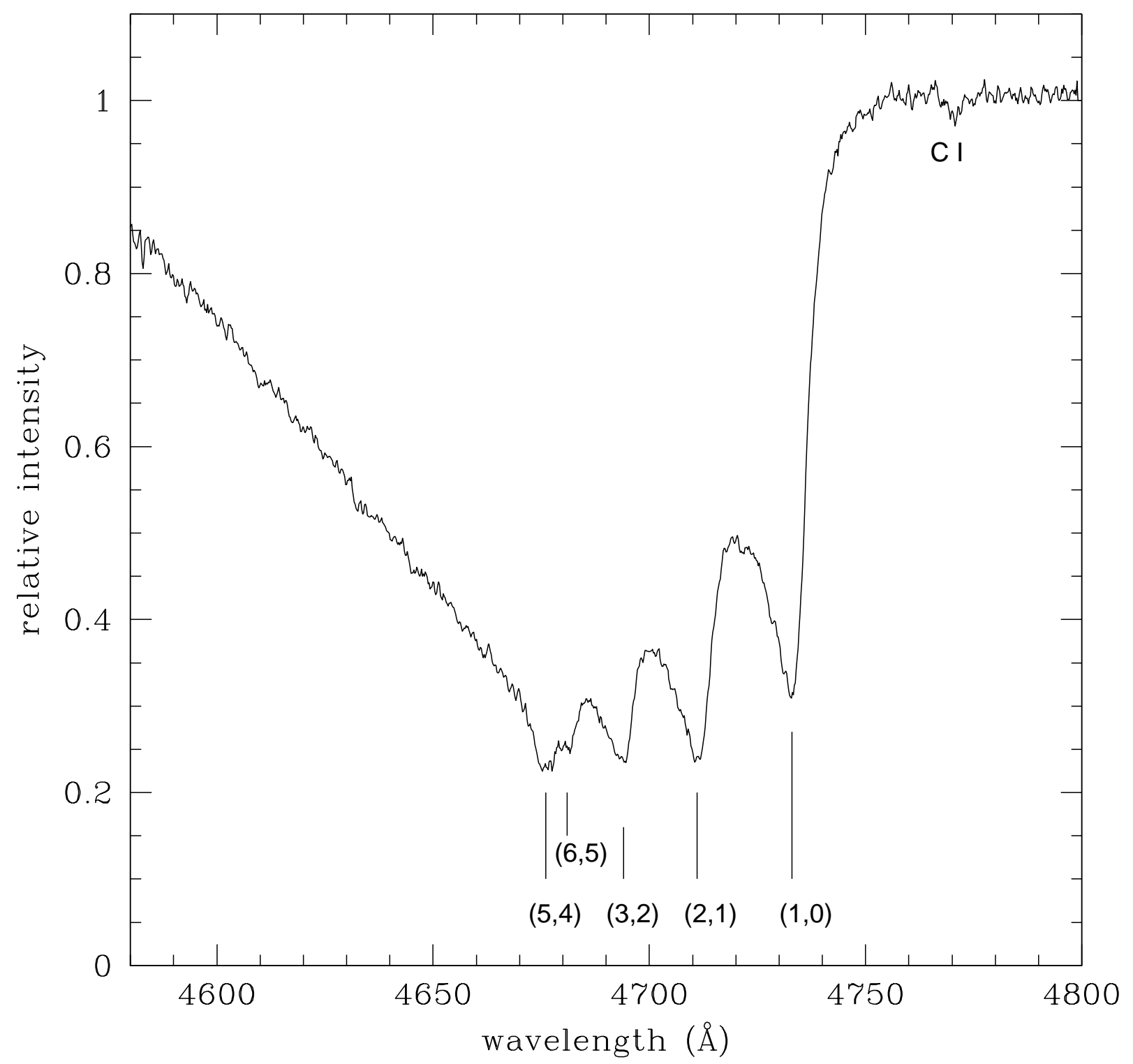

Fig. 2.- Portion of SALT spectra of BPM 27606 showing the main Swan band with $\Delta v=1$ of $C_{2}$ and the $\mathrm{C}$ I line near $4700 \AA$. 


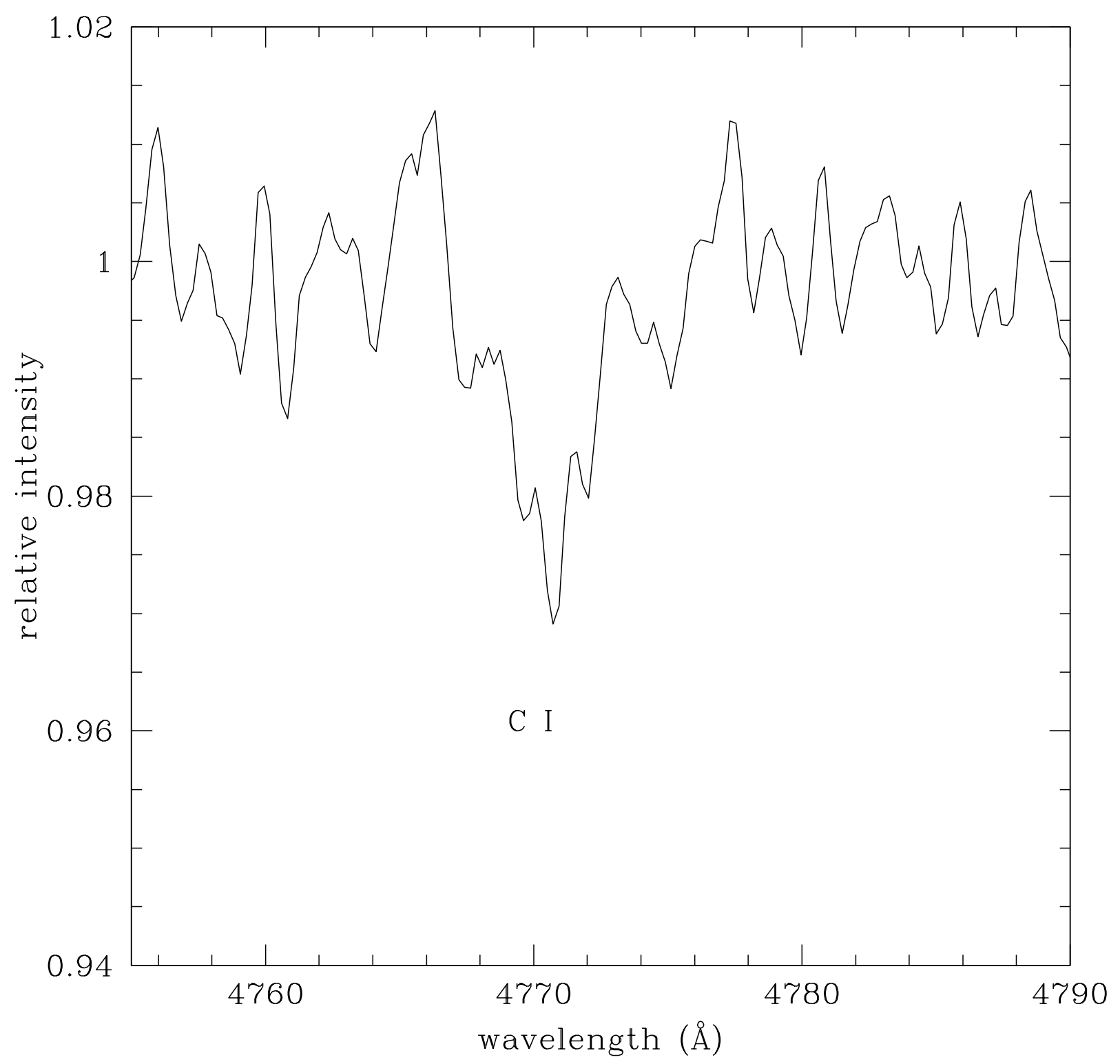

Fig. 3.- Enlarged portion of the spectrum of BPM 27606 showing the neutral carbon line near $4770 \AA$. 


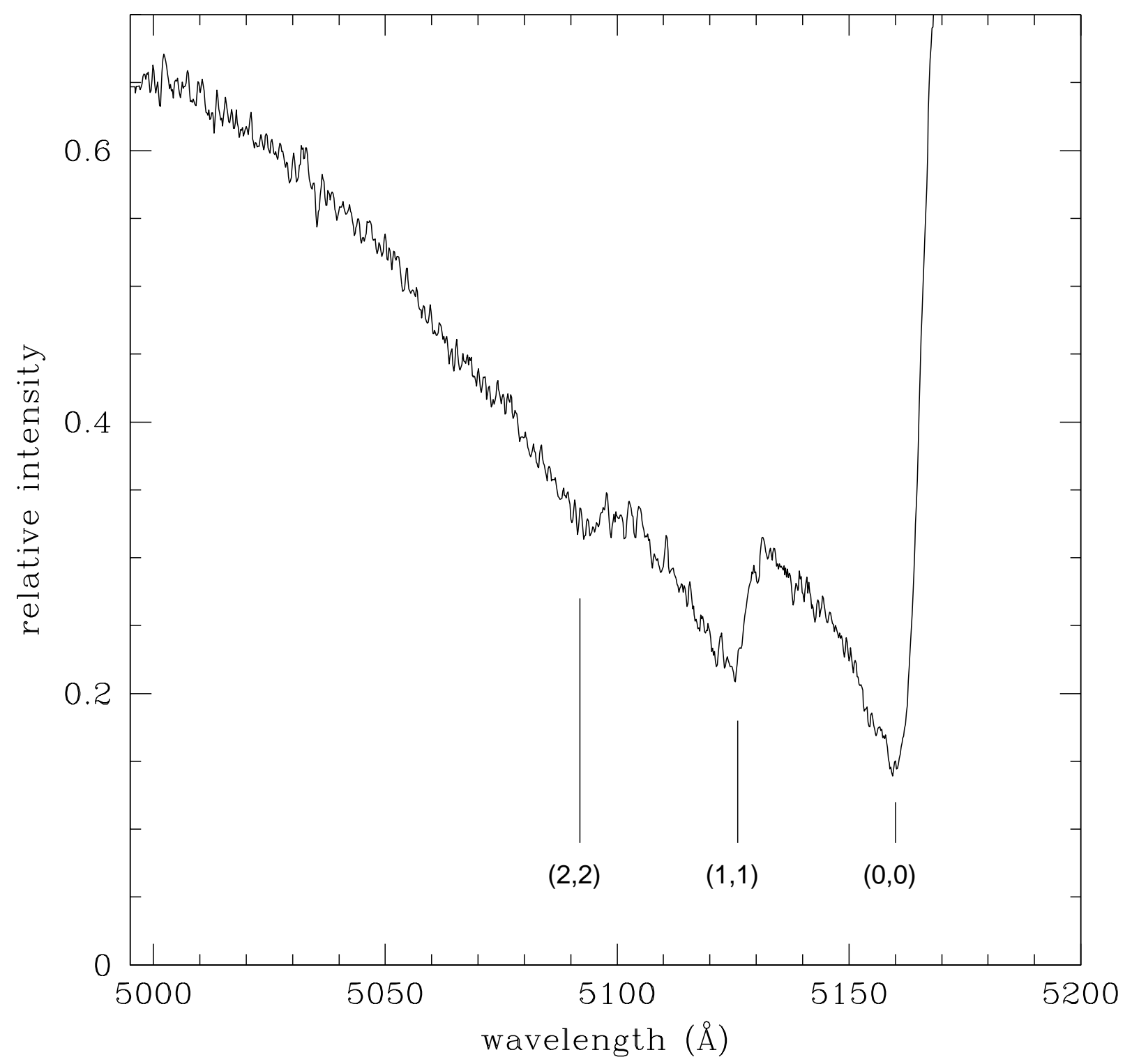

Fig. 4.- Portion of SALT spectra of BPM 27606 showing the Swan band with $\Delta v=0$ of $C_{2}$ near $5100 \AA$. 


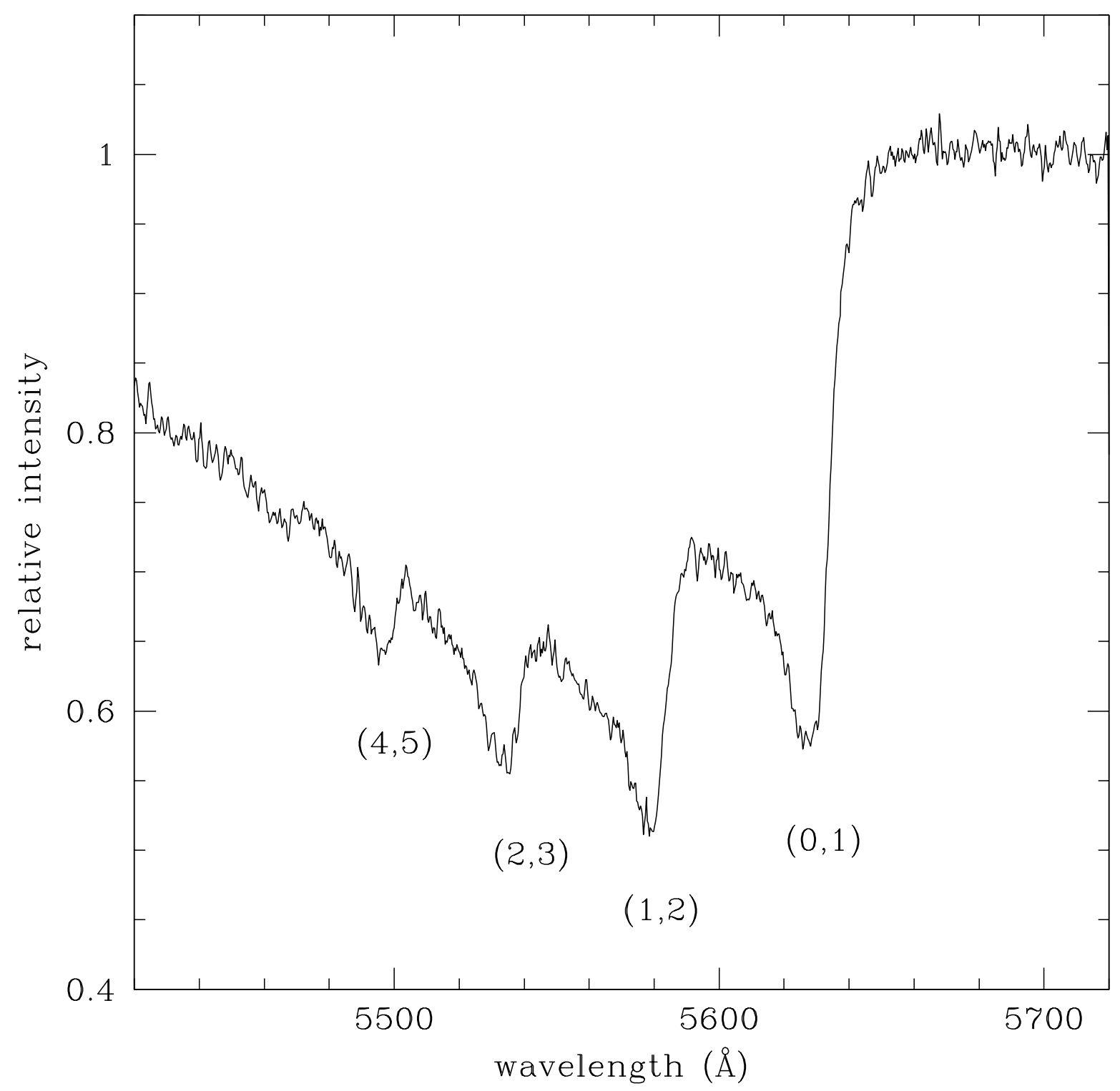

Fig. 5.- Portion of SALT spectra of BPM 27606 showing the Swan band with $\Delta v=-1$ of $C_{2}$ near $5550 \AA$. 


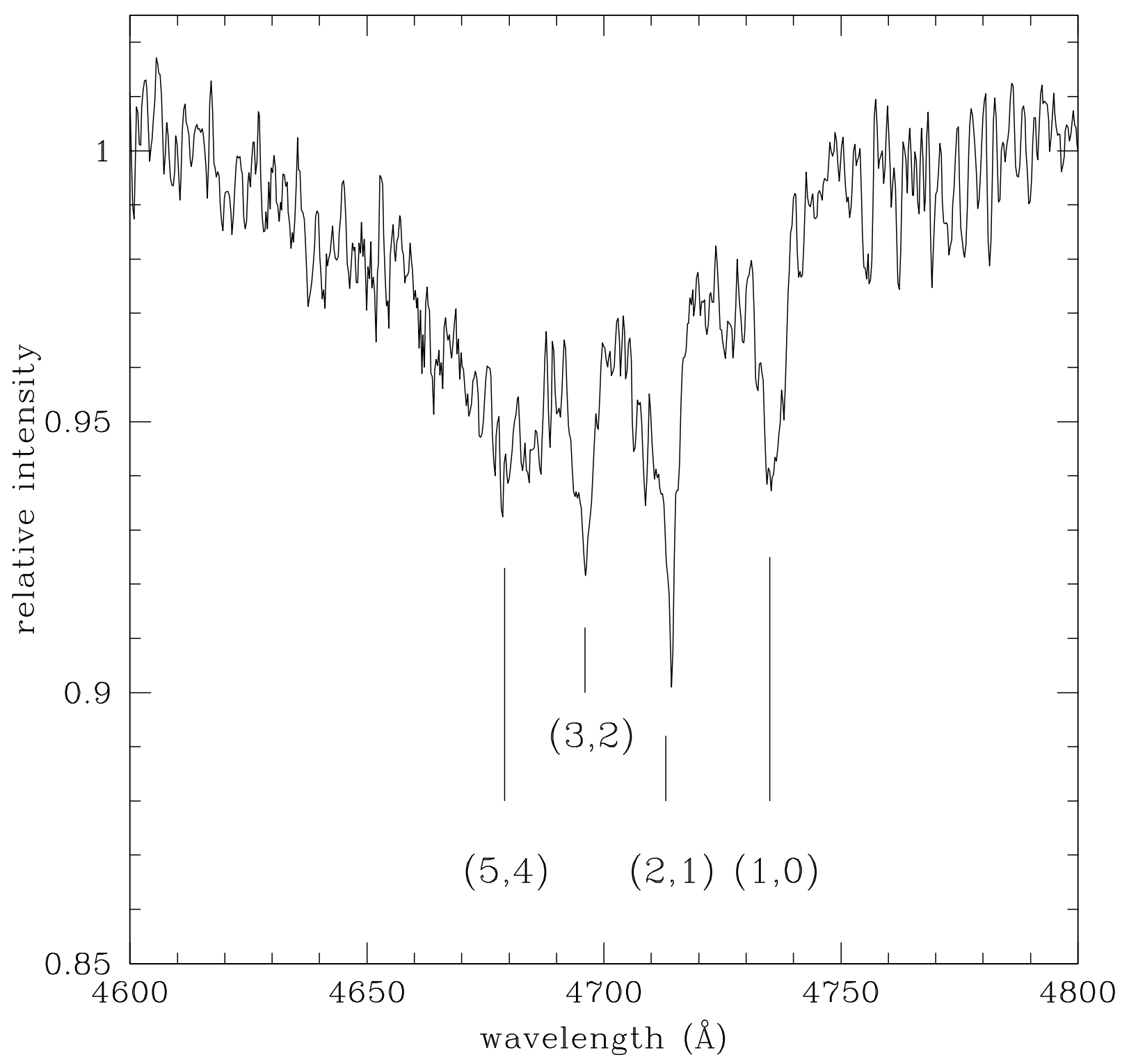

Fig. 6.- Portion of spectra of Procyon B showing the $\mathrm{C}_{2}$ Swan band around $4700 \AA$. 


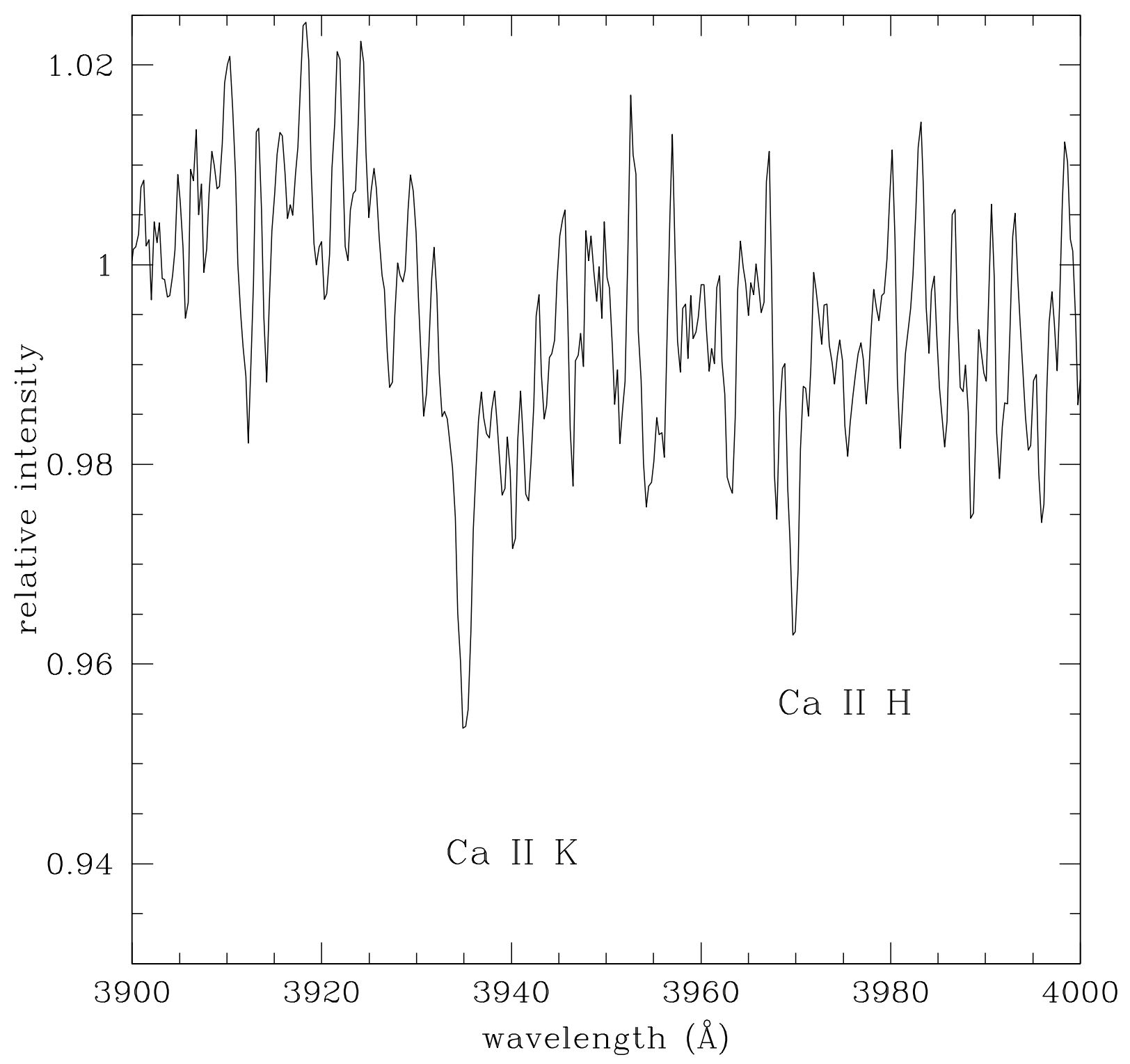

Fig. 7.- Portion of spectra of Procyon B showing the Ca II H and K lines. 